\title{
OPEN Systematic optimization of visible light-induced crosslinking conditions of gelatin methacryloyl (GelMA)
}

\author{
Sina Sharifi ${ }^{1 凶}$, Hannah Sharifi ${ }^{1}$, Ali Akbari ${ }^{2}$ \& James Chodosh ${ }^{1}$
}

Gelatin methacryloyl (GelMA) is one of the most widely used photo-crosslinkable biopolymers in tissue engineering. In in presence of an appropriate photoinitiator, the light activation triggers the crosslinking process, which provides shape fidelity and stability at physiological temperature. Although ultraviolet (UV) has been extensively explored for photo-crosslinking, its application has been linked to numerous biosafety concerns, originated from UV phototoxicity. Eosin Y, in combination with TEOA and VC, is a biosafe photoinitiation system that can be activated via visible light instead of UV and bypasses those biosafety concerns; however, the crosslinking system needs fine-tuning and optimization. In order to systematically optimize the crosslinking conditions, we herein independently varied the concentrations of Eosin $Y[(E Y)]$, triethanolamine (TEOA), vinyl caprolactam (VC), GelMA precursor, and crosslinking times and assessed the effect of those parameters on the properties the hydrogel. Our data showed that except EY, which exhibited an optimal concentration ( $0.05 \mathrm{mM})$, increasing [TEOA], [VA], [GelMA], or crosslinking time improved mechanical (tensile strength/modulus and compressive modulus), adhesion (lap shear strength), swelling, biodegradation properties of the hydrogel. However, increasing the concentrations of crosslinking reagents ([TEOA], [VA], [GelMA]) reduced cell viability in 3-dimensional (3D) cell culture. This study enabled us to optimize the crosslinking conditions to improve the properties of the GelMA hydrogel and to generate a library of hydrogels with defined properties essential for different biomedical applications.

Protein-based hydrogels including collagen, fibrin, elastin, silk, and gelatin have been extensively used in various biomedical applications. These materials are generally biocompatible, and because of their innate characteristics, can modulate cellular adhesion, proliferation, migration, and differentiation ${ }^{1-4}$. Gelatin methacryloyl (GelMA) is one of the most studied and versatile biopolymers to form a hydrogel used in biomedical engineering studies, such as micropatterning, fluidic systems, 3-D scaffolds, bioprinting with different cell types, tissue adhesives, and drug delivery ${ }^{3,5}$. GelMA mimics many important properties of native extracellular matrix (ECM), contains cell binding (e.g. Arg-Gly-Asp (RGD)) and matrix metalloproteinase (MMP)-sensitive degradation sites, and is biocompatible and tunable ${ }^{6-8}$. Taking into account the burgeoning interest in biopolymer-based hydrogels and the potential of GelMA, optimizing the efficacy of the preparation process ${ }^{5,9}$ and crosslinking are important.

To form a covalently crosslinked hydrogel network, the GelMA precursor undergoes a radical polymerization in the presence of a photoinitiator and light with an appropriate wavelength and intensity ${ }^{5}$. Ultraviolet (UV) is the most commonly used light to photo-crosslink GelMA and similar methacrylated materials such as tropoelastin ${ }^{10}$, elastin-like polypeptides ${ }^{11}$, poly(ethylene glycol) (PEG)-diacrylate hydrogels ${ }^{12}$, and many other elastomers ${ }^{13,14}$. However, there are several biosafety concerns associated with the application of UV light when it comes to using living cells and tissues. It was shown that UV light can cause damage either through pyrimidine dimerization or generation of reactive oxygen species (ROS) that may induce oxidative damage to DNA and lead to accelerated tissue aging, immunosuppression, and ultimately to cancer ${ }^{15-17}$. The combination of UV light and common UV photoinitiator (Irgacure 2959) also was shown to have harmful effects on cell viability, correlated with the

${ }^{1}$ Disruptive Technology Laboratory, Department of Ophthalmology, Harvard Medical School, Massachusetts Eye and Ear and Schepens Eye Research Institute, Boston, MA, USA. ${ }^{2}$ Solid Tumor Research Center, Research Institute for Cellular and Molecular Medicine, Urmia University of Medical Sciences, Urmia, Iran. ${ }^{\boxplus}$ email: Sina_sharifi@ meei.harvard.edu 
irradiation time ${ }^{18,19}$. Besides such phototoxic effects, UV light has limited penetration to tissues and hydrogels, which adds an additional hurdle to its biomedical applications. These challenges have propelled the scientific community to explore the application of light with higher wavelengths including visible light in conjunction with a photoinitiator that can be activated in those wavelength $\mathrm{s}^{20-22}$. Various visible-light photoinitiation systems, including lithium phenyl-2,4,6-trimethylbenzoylphosphinate (LAP) (absorption peak of $405 \mathrm{~nm}$ ), riboflavin (absorption peak of 440 and $371 \mathrm{~nm}$ ), carboxylated camphorquinone, (absorption peak of $470 \mathrm{~nm}$ ), and Eosin Y (EY) (absorption peak of $510 \mathrm{~nm}$ ) have been explored in biomedical applications ${ }^{23}$. The initiation systems that use higher wavelengths (e.g. green light) for activation are generally safer than those with lower wavelength energy, as the energy of photons used for crosslinking has a direct correlation with its phototoxicity ${ }^{24}$. For example, LAP is a single-component initiation system with high thermal stability and good solubility and is colorless; however, it is activated by blue visible light, which was shown to have some level of toxicity ${ }^{25}$. Eosin Y (EY) system, on the other hand, is a two-component initiation system, in which EY acts as photosensitizer and triethanolamine (TEOA) acts as an initiator and is activated by visible light with higher wavelengths to generate free radicals for the polymerization reaction ${ }^{26-28}$. The resultant hydrogel is optically transparent and has a pale yellow color, which originates from the yellow color of the activated EY. To accelerate gelation kinetics, different co-monomers such as N-Vinylcaprolactam (VC) or 1-vinyl-2 pyrrolidinone (NVP) are usually added to the crosslinking solution ${ }^{4,29}$. EY system is also Food and Drug Administration (FDA)-approved for the application of photo-crosslinkable lung sealant FocalSeal ${ }^{\oplus}$ (Genzyme Biosurgical, Cambridge, MA). The EY system can bypass phototoxicity induced by UV light, but the chemical reagents used in the crosslinking system (EY, TEOA, and VC) are toxic in high concentrations-especially for cellular photoencapsulation, which has a wide range of applications including 3-D bioprinting, differentiation studies, drug discovery and pharmacological applications, cancer research, gene, and protein expression studies ${ }^{30-33}$. To understand how altering the crosslinking conditions impact properties of the hydrogel, we herein performed a systematic study in which we independently varied the concentration of crosslinking reagents (EY, TEOA, and VC), the concentration of GelMA precursor, and the crosslinking time and studied the resulting changes in chemical, physical, mechanical, and biological properties of the hydrogel.

\section{Results and discussion}

Tuning Eosin $\mathrm{Y}$ concentration. EY is a photosensitizer, used in combination with the TEOA (initiator) and VC (co-monomer) for photo-induced crosslinking reaction under visible light ${ }^{28}$. When the EY absorption overlaps with the irradiated light spectrum, EY absorbs the light and moves to an excited state. Excited EY then abstracts a hydrogen radical $\left(\mathrm{H}^{*}\right)$ from TEOA and generates a TEOA radical, which serves as an initiator of crosslinking ${ }^{26-28,34}$. Visible light-induced crosslinking often necessitates the addition of co-monomers, such as N-Vinylcaprolactam (VC) or 1-vinyl-2 pyrrolidinone (NVP) to accelerate gelation kinetics. The addition of a co-monomer has several impacts: (1) it increases the vinyl group concentration in the reaction media to synergistically cross-propagate with methacrylate groups of GelMA; (2) it expedites a diffusion of radicalized compounds in the reaction media ${ }^{35}$; and (3) it facilitates free-radical polymerization through prevention of the scavenging the free-radicals by available oxygen ${ }^{12,36}$. These enhance the crosslinking density of the polymeric network and subsequently improve the structural and mechanical properties of the hydrogel ${ }^{37}$. Therefore, the crosslinking conditions are a valuable tool to control the physiochemical and biological characteristics of the hydrogel ${ }^{26,35-41}$. While extensive studies have been performed to understand the effects of polymerization conditions on the properties of polyethylene glycol (PEG)-based hydrogels ${ }^{39,40,42,43}$, limited reports have focused on the effects of polymerization conditions of the GelMA hydrogel. Noshadi et al. varied [TEOA] from 0.5 to $1.5 \%$ $(\mathrm{v} / \mathrm{v})$ and [VC] from 0.5 to $1.5 \%(\mathrm{w} / \mathrm{v})$ while retaining [EY] at $0.1 \mathrm{mM}$, and studied the mechanical properties of crosslinked hydrogels under the visible light irradiation ${ }^{26}$. They found that the compressive and tensile moduli of the GelMA hydrogels $(10 \% \mathrm{w} / \mathrm{v})$ were directly correlated with [VC] and [TEOA], varying from 5-56.5 kPa, and 5 to $22.7 \mathrm{kPa}$, respectively. Recently, a modification in the crosslinking condition ( [TEOA] $=1.875 \%(\mathrm{w} / \mathrm{v}$ ), $[\mathrm{VC}]=1.25 \%(\mathrm{w} / \mathrm{v})$, and $[\mathrm{EY}]=0.5 \mathrm{mM})$ was shown to increase the elastic modulus and tensile strength up to $224.4 \pm 32.3$ and $45.3 \pm 4.1 \mathrm{kPa}$, respectively when [GelMA precursor] is $20 \%(\mathrm{w} / \mathrm{v})$ after 4 min irradiation with visible light (450-550 $\mathrm{nm}$, and the intensity of $\left.100 \mathrm{~mW} / \mathrm{cm}^{2}\right)^{22}$.

To better understand how crosslinking conditions impact hydrogel properties, we herein performed a comprehensive systematic study and varied the concentration of crosslinking reagents in a wider range $([\mathrm{EY}]=0.005-1 \mathrm{mM} ;[\mathrm{TEOA}]=0.05-5 \%(\mathrm{w} / \mathrm{v}) ;[\mathrm{VC}]=0.05-5 \%(\mathrm{w} / \mathrm{v}) ;[$ GelMA precursor $]=5-20 \%)$; and time $=0.5=10 \mathrm{~min})($ Table $\mathrm{S} 1)$ than in prior work.

Successful polymerization reaction necessitates the generation of radical species with optimal concentrations. Such optimal concentrations assure that the reaction is not slow ([radical species] is low), or the reaction mixture does not contain too many free radicals in a close proximity, which can lead to smaller molecular weight chains ([radical species] is high). To generate those radical species, both EY and TEOA are necessary. Lower $[E Y]$ in the reaction leads to lower [activated EY], and lower [TEOA], and consequently fewer radical species, and having higher [EY] results in the opposite. Therefore, [radical species] can be approximately correlated with [EY]. However, at given [EY], if [TEOA] is too low, then those activated EY species are unable to activate TEOA to generate radicals, and if [TEOA] is too high, then the extra TEOA will remain unused. These indicate that the impact of EY is primary to that of TEOA. On that basis, we first tuned [EY] and then [TEOA]. Inspired by previous studies ${ }^{5,22,26,28}$, we selected [EY] from 0.005 to $1 \mathrm{mM}(0.005,0.01,0.02,0.05,0.1,0.2,0.5,1 \mathrm{mM})$, while maintaining the other parameters constant $([\mathrm{TEOA}]=1 \%(\mathrm{w} / \mathrm{v}) ;[\mathrm{VC}]=0.5 \%(\mathrm{w} / \mathrm{v}) ;[$ GelMA precursor $]=20 \%$ $(\mathrm{w} / \mathrm{v})$; and time $=1 \mathrm{~min})$. Our studies show that the tensile strength and elastic modulus of GelMA are strongly dependent on [EY] (Fig. 1a-c,). While at lower [EY] $(<0.05 \mathrm{mM})$, there was a direct correlation between [EY] and tensile strength or modulus, at higher [EY] $(>0.05 \mathrm{mM})$, there was an inverse correlation (Fig. 1a-c \& Table S2). The former could originate from incomplete crosslinking of the polymeric network because of lack of 



Figure 1. Tuning [EY] and its impact on the structural and biological properties of GelMA. a) Representative tensile stress/strain plots for GelMA ( $20 \% \mathrm{w} / \mathrm{v}$ and crosslinked for $1 \mathrm{~min}$ ) with varying [EY] and corresponding average tensile modulus (b) and tensile strength (c), along with mean compressive modulus (d), and shear strength (e) as a function of [EY]. Collagenase induced degradation (f) and swelling ratio (g) of the GelMA hydrogels formed with varying [EY] $(20 \% \mathrm{w} / \mathrm{v})$ as a function of time, and corresponding swelling ratios (h) after $24 \mathrm{~h}$ of incubation at $37^{\circ} \mathrm{C}$. (i) Representative Live-Dead images of the encapsulated corneal fibroblasts (CF) in 3-dimensional (3-D) cell culture and the quantification of cell viability (j) after 1 day of post-seeding on GelMA hydrogels $(10 \% \mathrm{w} / \mathrm{v}$ and crosslinked for $1 \mathrm{~min})$ formed with varying [EY] (scale bars: $150 \mu \mathrm{m})$. Values are presented as mean $\pm \mathrm{SD} ; \mathrm{n}=4$. ns, ${ }^{*},{ }^{* *},{ }^{* * *}$, and ${ }^{* * *}$ represent $p>0.05, p \leq 0.05, p \leq 0.01, p \leq 0.001$ and $p \leq 0.0001$, respectively.

$\mathrm{EY}$, and consequently lack of radical initiator ${ }^{37}$. However, the latter could stem from the presence of too many radical species in the reaction media in close proximity, leading to recombination or disproportionation of those radicals and consequently to the termination ${ }^{44,45}$. These data suggest that there is an optimal concentration range of EY $(0.05 \mathrm{mM})$ to afford the highest tensile modulus and strength. The compression and lap shear measurements offer similar results, indicating that under optimal [EY], the compression modulus and shear strength of hydrogel can be improved up to $160 \mathrm{kPa}$ and $560 \mathrm{kPa}$, respectively only after $1 \mathrm{~min}$ of crosslinking using a white LED light with the intensity of $20 \mathrm{~mW} / \mathrm{cm}^{2}$ (Fig. 1d-e).

To study the stability of the synthesized hydrogels, which is one of the key components to define its biomedical applications, we used the enzymatic degradation assay. We incubated the hydrogels in a solution containing collagenase and compared the dried weight of the samples to the initial dried weight as a function of exposure time $^{7}$. Consistent with the mechanical properties, hydrogels formed under the optimal $[\mathrm{EY}](\mathrm{ca} .=0.05 \mathrm{mM})$ had longer retentions (Fig. If \& Table S3). The enhanced stability against enzymatic degradation indicates an improved crosslinking density, which in turn can restrict the accessibility of the enzyme to the cleavage sites of the hydrogel while increasing the anchoring points of the network ${ }^{46}$.

The swelling ratio as a function of time shows a minima at $(\mathrm{EY}=0.05 \mathrm{mM})$ (Fig. $1 \mathrm{~g}, \mathrm{~h}$ \& Table S4). This behavior is consistent with the prior mechanical evaluations and is likely originated from varying crosslinking degrees. We then studied the cytotoxicity of crosslinking conditions on corneal fibroblasts in 3-D cell culture. Quantification of cellular viability, determined by the Live-Dead study, showed an inverse correlation between cell viability and [EY] (Fig. 1i, j). In the lower [EY] $(<0.05 \mathrm{mM})$ the difference in the cellular viability was not substantial. However, as [EY] was increased, its impact on the viability of the cells became more pronounced.

TuningTEOA concentration. After determining the optimum concentration range of EY $([\mathrm{EY}]=0.05 \mathrm{mM})$, which afforded the highest tensile modulus and strength, we retained that concentration and tuned [photoini- 

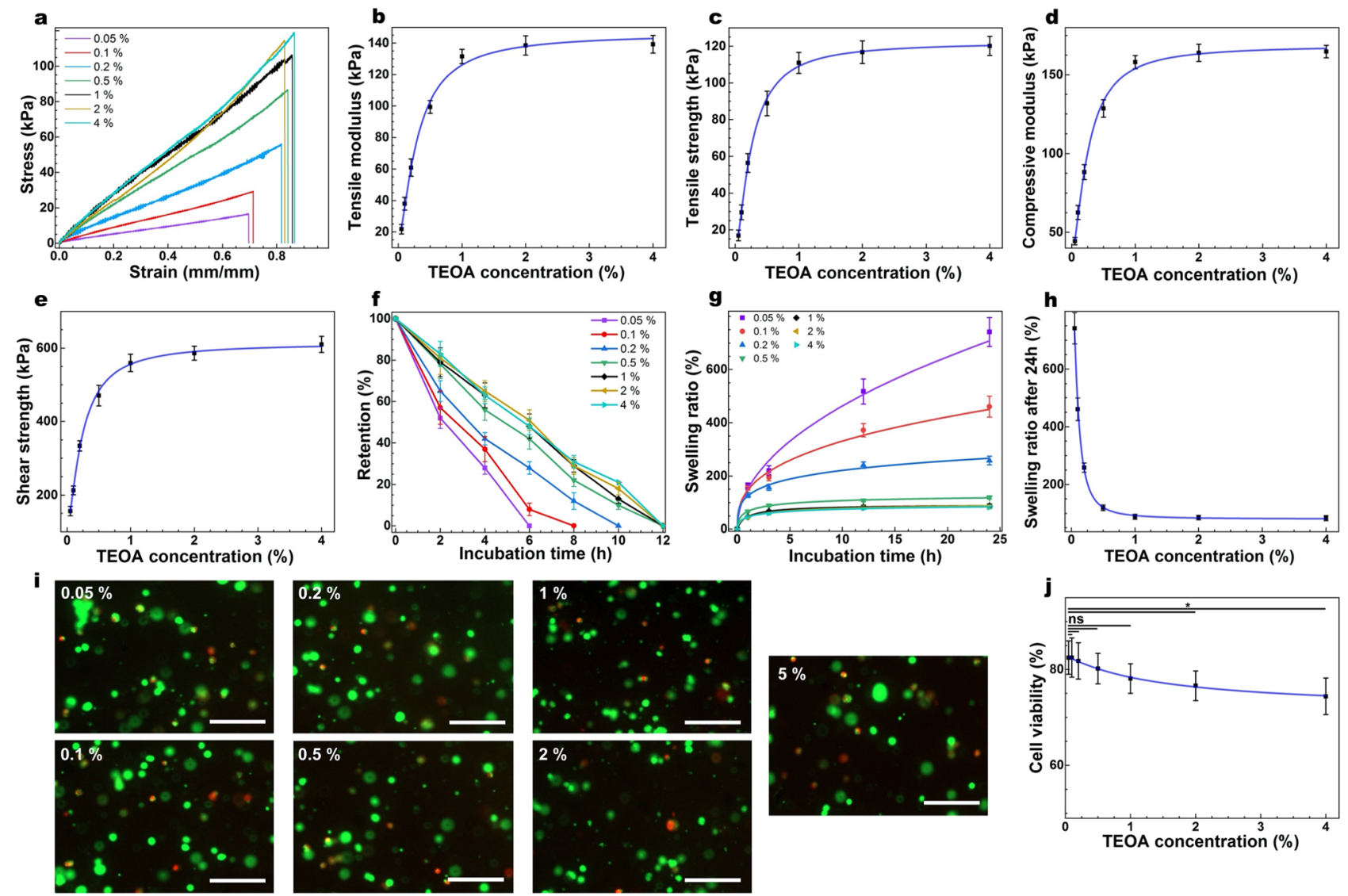

Figure 2. Tuning [TEOA] and its impact on the structural and biological properties of GelMA.(a) Representative tensile stress/strain plots for GelMA ( $20 \% \mathrm{w} / \mathrm{v}$ and crosslinked for $1 \mathrm{~min}$ ) with varying [TEOA] and their corresponding average tensile modulus (b) and tensile strength (c), along with mean compressive modulus (d), and shear strength (e) as a function of [TEOA]. Collagenase induced degradation (f) and swelling ratio (g) of the GelMA hydrogels formed with varying [TEOA] (20\% w/v) as a function of time and corresponding swelling ratios after $24 \mathrm{~h}$ of incubation at $37^{\circ} \mathrm{C}$. Representative Live-Dead images of the encapsulated CF in 3-D cell culture (i) and the quantification of cell viability $(\mathbf{j})$ after 1 day of post-seeding on GelMA hydrogels ( $10 \% \mathrm{w} / \mathrm{v}$ and crosslinked for $1 \mathrm{~min})$ formed with varying [TEOA] (scale bars: $150 \mu \mathrm{m}$ ). Values are presented as mean $\pm \mathrm{SD} ; \mathrm{n}=4$. ns and ${ }^{*}$, represent $p>0.05$ and $p \leq 0.05$, respectively.

tiator (TEOA)] from 0.05 to $4 \%(\mathrm{w} / \mathrm{v})(0.05,0.1,0.2,0.5,1,2,4 \%)$ and studied mechanical, structural, and degradation properties of the hydrogels along with the cytotoxicity of the reaction (Table S1). As shown in Fig. $2 \mathrm{a}-\mathrm{c}$, there was a sigmoidal correlation between tensile strength/modulus and [TEOA], ranging from 17 to $120 \mathrm{kPa}$, and $22-140 \mathrm{kPa}$, respectively (Table S2). At lower [TEOA] $(0.05-0.5 \%(\mathrm{w} / \mathrm{v}))$, the tensile properties were strongly correlated with [TEOA], indicating lack of initiator at lower [TEOA], slower polymerization kinetics, and incomplete crosslinking at a given time ${ }^{45}$. However, such dependence plateaued at the higher [TEOA] $(>1 \%(\mathrm{w} / \mathrm{v}))$, suggesting that excess TEOA may not be converted to radical form and consequently may not contribute to the crosslinking reaction as the excited EY limits the radical form of TEOA ${ }^{44}$. The compression modulus and shear strength as a function of [TEOA] showed similar trends, ranging from 44 to $164 \mathrm{kPa}$ and 156-610 kPa, respectively (Fig. 2d, e). The retention of the hydrogel in the collagenase solution was also directly correlated with [TEOA], with the fastest degradation in the hydrogels formed with [TOEA] $=0.05 \%(6 \mathrm{~h})$ and slowest for the hydrogels formed with [TOEA] $>1 \%$ (Fig. $2 \mathrm{f} \&$ Table S3). The swelling ratio, in contrast, exhibited an inverse sigmoidal correlation with [TEOA] ranging from 83 to $741 \%$ after $24 \mathrm{~h}$ of incubation in the PBS at room temperature (Fig. $2 \mathrm{~g}$, h \& Table S4). At lower [TEOA] $=0.05-1 \%(\mathrm{w} / \mathrm{v})$, the correlation between swelling ratio and $[$ TEOA] was strong, and then plateaued at higher $[$ TEOA] $>1 \%(\mathrm{w} / \mathrm{v})$. These data agree with mechanical properties assessment and are believed to originate from varying crosslinking degrees between polymeric chains ${ }^{47,48}$.

Cytotoxicity studies also showed an inverse sigmoidal correlation between cell viability and [TEOA] (Fig. 2i, $\mathrm{j})$, demonstrating that for lower [TEOA] $(<0.05 \mathrm{mM})$, cellular viability was high, yet as [TEOA] was increased, the cellular viability slowly dropped. Such decline viability indicates the toxicity of TEOA at higher concentrations as previously shown 28,49 .

Tuning VC concentration. VC functions as a co-monomer to accelerate the reaction rate and increase the crosslinking density. Without VC the crosslinking can happen, yet with a much slower rate and affords a hydrogel with inferior mechanical properties. Therefore, VC presence is not as vital as EY and TEOA. After finding the 

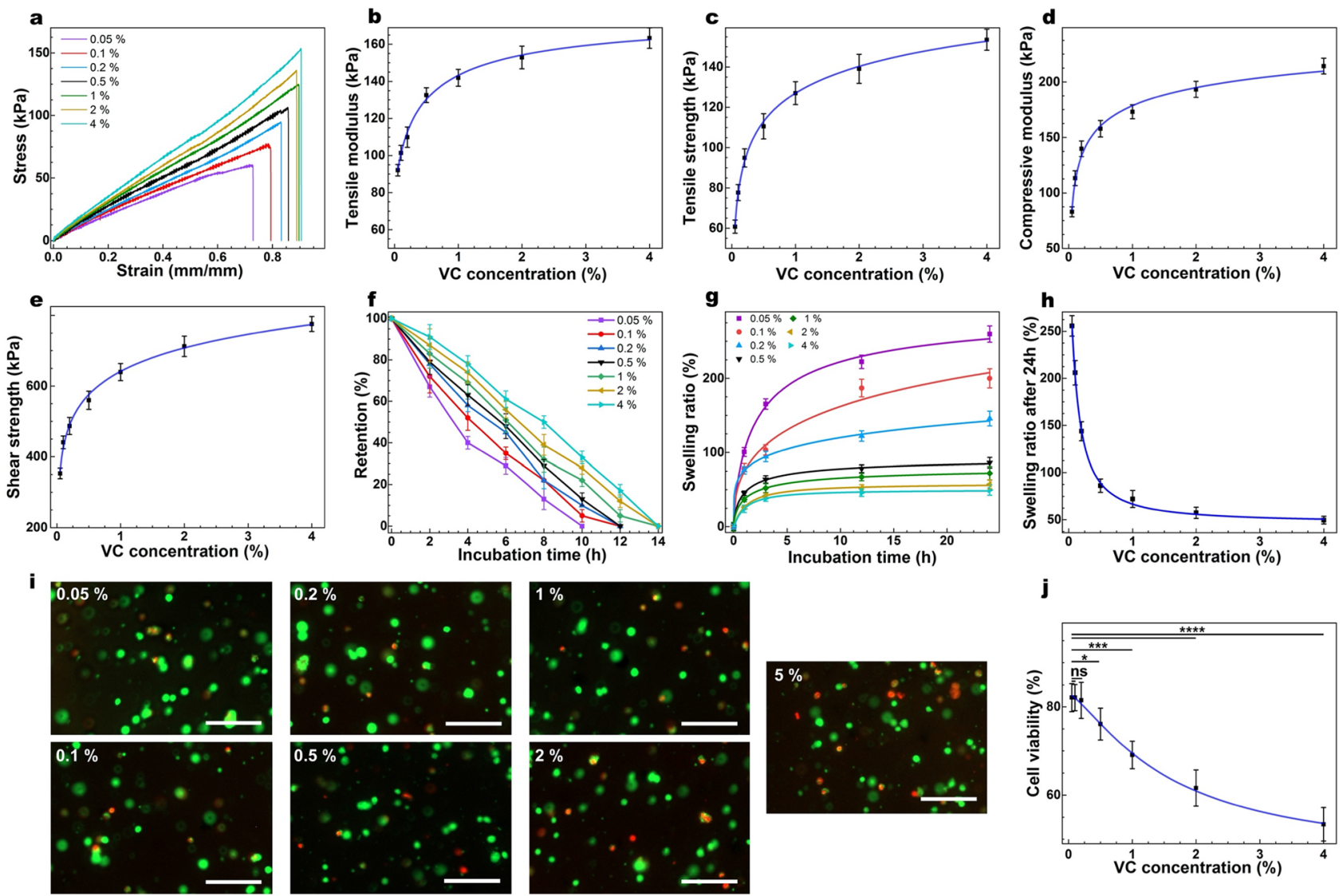

Figure 3. Tuning [VC] and its impact on the structural and biological properties of GelMA. (a) Representative tensile stress/strain plots for GelMA ( $20 \% \mathrm{w} / \mathrm{v}$ and crosslinked for $1 \mathrm{~min})$ with varying [VC] and their corresponding average tensile modulus (b) and tensile strength (c), along with mean compressive modulus (d), and shear strength (e) as a function of [VC]. Collagenase induced degradation (f) and swelling ratio (g) of the GelMA hydrogels formed with varying [VC] $(20 \% \mathrm{w} / \mathrm{v})$ as a function of time, and corresponding swelling ratios after $24 \mathrm{~h}$ of incubation at $37^{\circ} \mathrm{C}$. Representative Live-Dead images of the encapsulated CF in 3-D cell culture (i) and the quantification of cell viability (j) after 1 day of post-seeding on GelMA hydrogels (10\% w/v and crosslinked for $1 \mathrm{~min}$ ) formed with varying [VC] (scale bars: $150 \mu \mathrm{m}$ ). Values are presented as mean $\pm \mathrm{SD} ; \mathrm{n}=4$. $\mathrm{ns},{ }^{*},{ }^{* *},{ }^{* *}$, and ${ }^{* * *}$ represent $p>0.05, p \leq 0.05, p \leq 0.01, p \leq 0.001$ and $p \leq 0.0001$, respectively.

optimal [EY] $(0.05 \mathrm{mM})$ and [TEOA] (1\% (w/v) highest efficiency before the presence of a plateau), we maintained the concentrations of those crosslinking agents and examined the mechanical, structural, and degradation properties of the hydrogel and their cytotoxicity (Table S1) as a function of [co-monomer (VC)] $(0.05,0.1,0.2$, $0.5,1,2,4 \%)$. As illustrated in Fig. 3a-c and Table S2, there is a sigmoidal correlation between tensile strength/ modulus and [VC]. Tensile strength/modulus ranged from $60-153 \mathrm{kPa}$, and 92-164 kPa, respectively. At lower [VC], the tensile properties appeared to correlate strongly with [VC], suggesting the importance of the comonomer in the crosslinking reaction. Such importance is likely because the addition of co-monomer increases the vinyl group abundance in the reaction, which can synergistically cross-propagate with methacrylate groups of GelMA while enhancing the diffusion of radicalized species ${ }^{27,35}$ and facilitating free-radical polymerization via preventing the scavenging free-radicals by oxygen ${ }^{12,36}$. On the other hand, at the higher [VC] $(>1 \%(\mathrm{w} / \mathrm{v}))$ the correlation between tensile properties and [VC] was comparatively less pronounced. This correlation pattern suggests that excess [VC] in the reaction mixture may function only as a co-monomer to cross-propagate with methacrylate groups of GelMA and thus increase the polymerization kinetics ${ }^{44,50}$. The compression and lap modulus as a function of [VC] showed similar trends, with those ranging from 102-214 $\mathrm{kPa}$ and $353-715 \mathrm{kPa}$, respectively (Fig. 3d, e \& Table S2). Therefore, [VC] can be a valuable tool to further enhance the mechanical properties of the desired hydrogel.

Degradation studies showed that the retention of the hydrogel in the collagenase solution was directly correlated with [VC], with the fastest degradation occurring in the hydrogels formed with [VC] $=0.05 \%(\mathrm{ca} .10 \mathrm{~h})$, and slowest degradation in those formed with $[\mathrm{VC}]>1 \%$ (Fig. $3 \mathrm{f} \&$ Table S3). The swelling test showed an inverse sigmoidal correlation between the swelling ratio and [VC] (Fig. 3g, h), spanning from 50 to $255 \%$ after $24 \mathrm{~h}$ of incubation in PBS. This data agrees with the mechanical properties, indicating lower swelling for the hydrogels with superior mechanical properties $([\mathrm{VC}]>1 \%(\mathrm{w} / \mathrm{v}))$, and higher for the hydrogels with inferior properties $([\mathrm{VC}]<0.5 \%(\mathrm{w} / \mathrm{v}))$ (Table S4). Higher [VC], which can co-crosslink with GelMA, can decrease the hydrophilicity of the hydrogel, which may contribute to the declined swelling ratio of the hydrogel ${ }^{51}$. The viability of corneal 
$\mathbf{a}$
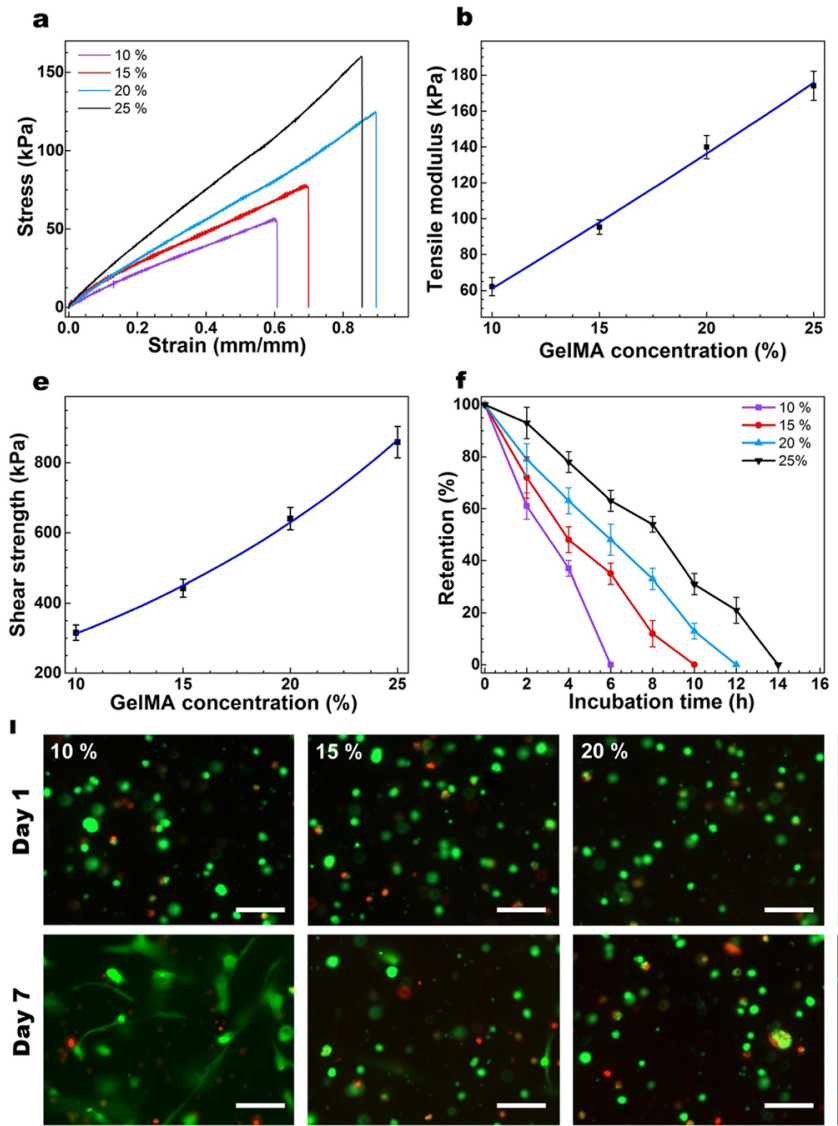
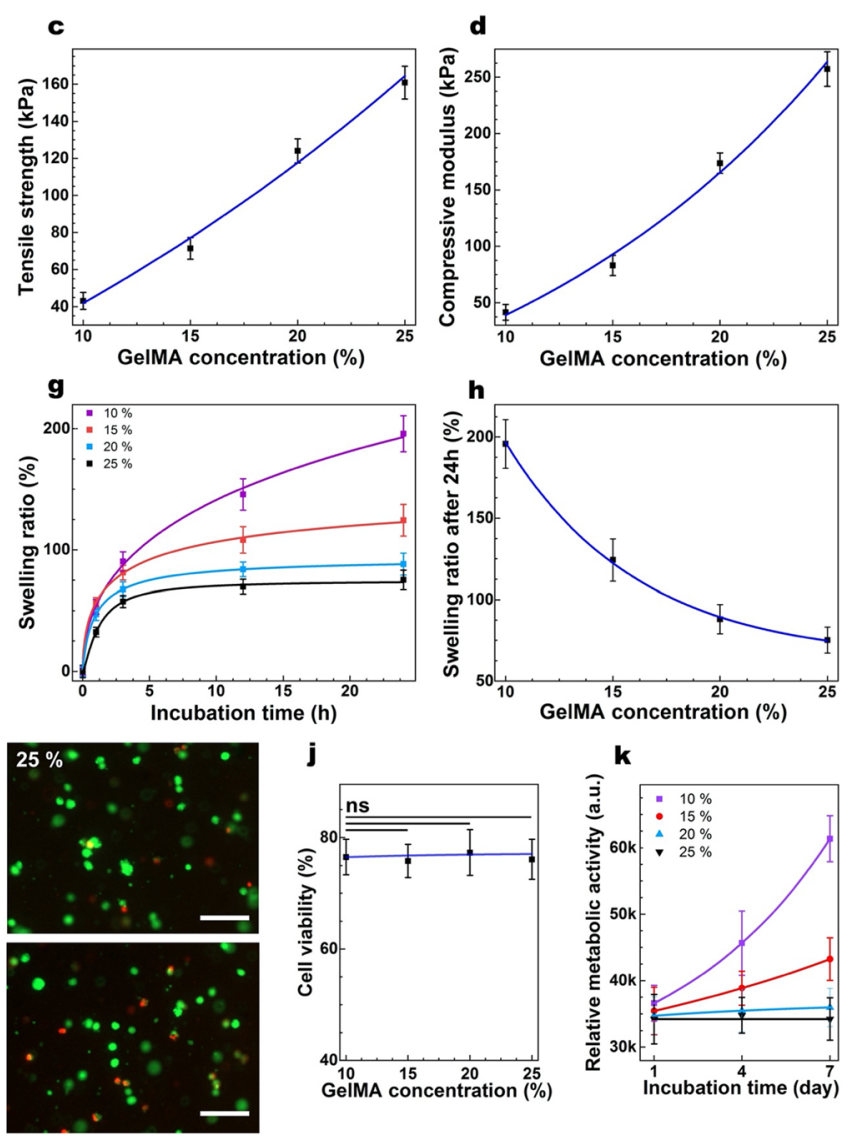

j
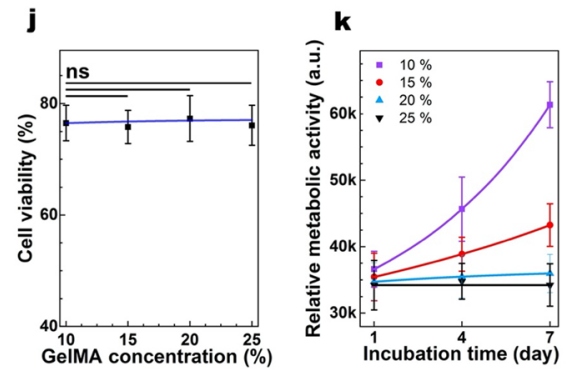

Figure 4. Tuning [GelMA precursor] and its impact on the structural and biological properties of GelMA. (a) Representative tensile stress/strain plots for GelMA (crosslinked for $1 \mathrm{~min}$ ) with varying concentrations and their corresponding average tensile modulus (b) and tensile strength (c), along with mean compressive modulus (d), and shear strength (e) as a function of [GelMA precursor]. Collagenase-induced degradation (f) and swelling ratio ( $\mathbf{g}$ ) of the GelMA hydrogels formed with varying concentrations as a function of time, and corresponding swelling ratios after $24 \mathrm{~h}$ of incubation at $37^{\circ} \mathrm{C}$. Representative Live-Dead images of the encapsulated CF in 3-D cell culture (i) and the quantification of cell viability (j) after 1 and 7 days of postseeding (crosslinked for $1 \mathrm{~min}$ ) formed with varying [GelMA] (scale bars: $150 \mu \mathrm{m}$ ). k) Quantification of metabolic activity of encapsulated CF on GelMA hydrogels formed with varying [GelMA] after 1, 4, and 7 days of post-seeding. Values are presented as mean $\pm S D ; n=4$. ns represents $p>0.05$.

fibroblasts showed an inverse sigmoidal correlation with [VC], suggesting the cytotoxicity of VC at higher concentrations (Fig. 3i, j) as previously shown ${ }^{28,52}$.

Tuning GelMA concentration. After finding the optimal [EY] (0.05 mM) and [TEOA] (1\% (w/v)) and [VC] $(1 \%(\mathrm{w} / \mathrm{v})$ highest efficiency before the presence of a plateau), we next retained those concentrations and varied the concentration of GelMA precursor from 10 to $25 \%(\mathrm{w} / \mathrm{v})(10,15,20,25 \%)$, and studied how physicochemical properties of the hydrogel behaved under such optimized conditions (Table S1). Tensile strength/ modulus as a function of [GelMA precursor] exhibited exponential growth correlations (Fig. 4a-c), with the tensile strength/modulus ranging from $57-161 \mathrm{kPa}$, and $62-181 \mathrm{kPa}$, respectively (Table S2). The higher the concentration of GelMA precursor, the higher the abundance of the vinyl-bearing precursor in the close vicinity, and the higher the chance for the propagation of generated radicals as opposed to termination to facilitate the gelation kinetics. Moreover, the hydrogels formed with a higher concentration of GelMA precursor are expected to have higher densities of the polymeric chains with lower porosities, and consequently superior mechanical properties. These explain the exponential dependence of the mechanical properties to [GelMA precursor] and suggest that through increasing precursor concentration, those properties can be significantly improved. The compression modulus and lap shear strength as a function of [GelMA precursor] exhibited similar trends and ranged from $93-245 \mathrm{kPa}$ and $315-859 \mathrm{kPa}$, respectively (Fig. 4d, e \& Table S2).

Enzymatic degradation testing showed the fastest degradation of the hydrogels formed with lower [GelMA precursor $]=10 \%(\mathrm{w} / \mathrm{v})$ with retention of $4 \mathrm{~h})$ and slowest degradation in the hydrogels formed with higher [GelMA precursor] $=25 \%$ (w/v) (Fig. 4f \& Table S3), with the retention of hydrogel in the collagenase solution for up to $14 \mathrm{~h}$. The swelling ratio assay indicated an exponential decay correlation between the swelling ratio and [GelMA precursor] (Fig. 4g, h \& Table S4) with a swelling ratio ranging from 75 to $195 \%$ after $24 \mathrm{~h}$ of incubation. 
$\mathbf{a}$


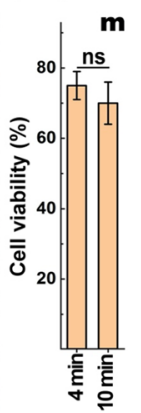

Figure 5. Tuning the crosslinking time and its impact on the structural and biological properties of GelMA. (a) Representative tensile stress/strain plots for GelMA $(20 \% \mathrm{w} / \mathrm{v})$ with varying crosslinking time and their corresponding average tensile modulus (b) and tensile strength (c), along with mean compressive modulus (d), and shear strength (e) as a function of crosslinking time. Collagenase induced degradation (f) and swelling ratio (g) of the GelMA hydrogels formed with varying crosslinking time $(20 \% \mathrm{w} / \mathrm{v})$ as a function of time, and corresponding swelling ratios after $24 \mathrm{~h}$ of incubation at $37^{\circ} \mathrm{C}$. Representative Live-Dead images of the encapsulated corneal CF in 3-D cell culture (i) and the quantification of cell viability after 1 day of post-seeding in GelMA hydrogels $(10 \% \mathrm{w} / \mathrm{v})$ with immediate washing $(\mathbf{j})$ and postponed washing $(\mathbf{k})$ all after 10 min formed with varying crosslinking time (scale bars: $150 \mu \mathrm{m}$ ). Representative Live-Dead images of the encapsulated mesenchymal stems cells in 3-D cell culture (l) and the quantification of cell viability after 1 day of post-seeding in GelMA hydrogels $(20 \% \mathrm{w} / \mathrm{v})$ crosslinked for $4 \& 10 \mathrm{~min}$. Values are presented as mean \pm SD. $n=4$; ns and * represent $p>0.05$ and $p \leq 0.05$, respectively.

This data is consistent with the mechanical properties and is believed to originate from varying porosity of the crosslinked hydrogel as previously shown ${ }^{5,26,41}$.

Cytotoxicity studies, on the other hand, did not show any correlation between cell viability and [GelMA precursor] (Fig. 4i, j). However, most of the encapsulated cells in the hydrogel formed with $10 \%$ (w/v) GelMA precursor elongated, spread, and formed interconnected networks with neighboring cells within 7 days of cell culture. Yet, the spreading was very limited in the hydrogel with $15 \%$ precursor as previously shown ${ }^{54}$, with no evidence of cellular spreading in the hydrogels formed with 20 and $25 \%$ of GelMA precursor (Fig. 4i). The metabolic activities of corneal fibroblasts as a function of incubation time showed a gradual increase, indicated by the relative fluorescence intensities for the hydrogels formed with 10 and 15\% GelMA concentrations, suggesting cellular growth and proliferation over time (Fig. 4k). Yet, the metabolic activities of the fibroblasts encapsulated within the hydrogel with a higher concentration of precursor did not change over time after 7 days of cell culture, indicating that higher [GelMA precursor] can slow cell growth and spreading because of enhanced structural and degradation properties of those hydrogels ${ }^{54}$. Yet, most of the encapsulated cells were viable, suggesting an appropriate level of permeability within the hydrogels network formed with a higher concentration of precursor.

Varying crosslinking time. To study the effect of crosslinking time on the physicochemical properties of the hydrogels, we next varied the visible light irradiation time from 0.5 to $10 \mathrm{~min}$, while maintaining the concentrations of crosslinking agents $([\mathrm{EY}]=0.05 \mathrm{mM},[\mathrm{TEOA}]=1 \%(\mathrm{w} / \mathrm{v}),[\mathrm{VC}]=1 \%(\mathrm{w} / \mathrm{v})$, and [GelMA precursor $]=20 \%(\mathrm{w} / \mathrm{v})($ Table S1). Tensile assessments indicated a sigmoidal correlation between crosslinking time and tensile modulus/strength (Fig. 5a-c), spanning from 49-181 kPa, and 56-164 kPa, respectively (Table S2). At the beginning of the visible light irradiation, the tensile properties were strongly correlated with the crosslink- 
ing time, suggesting the importance of irradiation on the generation of free radicals. However, such correlation gradually plateaued, particularly after 4 min of irradiation, suggesting that when the crosslinking reaction reaches the completion step, further irradiation may not significantly improve the mechanical properties. The compression and lap shear experiments exhibited similar trends with the compression modulus and shear strength spanning from $65-238 \mathrm{kPa}$ and $319-857 \mathrm{kPa}$, respectively (Fig. 5d, e \& Table S2).

Enzymatic degradation testing showed faster degradation of the hydrogels exposed to shorter light durations, and slower degradations for the hydrogels with longer exposures, with the retention time of 10-14 h in the collagenase solution (Fig. $5 \mathrm{f} \&$ Table S3). The swelling study showed an exponential decay correlation between the swelling ratio, which ranged from $25-189 \%$ after $24 \mathrm{~h}$ of incubation and crosslinking time (Fig. $5 \mathrm{~g}$, $\mathrm{h} \&$ Table S4). These data are consistent with the structural properties and indicate the importance of varying crosslinking degrees as previously shown ${ }^{54}$. Cytotoxicity studies suggested an inverse correlation between cell viability and crosslinking time (Fig. 5i, j). Such cytotoxicity could originate from prolonged exposure to the crosslinking agents during the gelation reaction. To normalize the effect of exposure to crosslinking agents, we generate a series of hydrogels with varying crosslinking time, yet with the same exposure time to the crosslinking reagents as they all were rinsed after $10 \mathrm{~min}$. Quantification of cellular viability shown by the Live-Dead assay suggested that similar cell viability across all groups (Fig. 5k). This data verifies that prolonged exposure to the crosslinking agents reduces cell viability and indicates the importance of the concentration of crosslinking reagents and exposure time in cell encapsulation studies. To understand how the optimized conditions impact the viability of other types of cells such as human mesenchymal stem cells (MSCs), which are the prominent subject in the modern research era due to their importance in tissue engineering and regenerative medicine $\mathrm{e}^{55}$, we photoencapsulated them in GelMA solution containing (20\% (w/v) GelMA, $0.05 \mathrm{mM}$ EY, 1\% (w/v) TEOA, and $1 \%(\mathrm{w} / \mathrm{v})$ VC. Cytotoxicity studies showed that MSCs have high viability $~ 75 \%$ and $72 \%$ after 4 and $10 \mathrm{~min}$ of light exposure (Fig. 5l, m). While we did not find a statistically significant difference between the viability of cells crosslinked for 4 and $10 \mathrm{~min}$, the general trend of declining viability as a function of exposure is evident. This data suggests that the crosslinking conditions have a similar impact on other types of cells.

Overall, in this report, we performed a systematic study of the impacts of tuning the concentrations of crosslinking reagents, [GelMA precursor], and crosslinking times, generated a library of GelMA hydrogels, and studied their corresponding chemical, physical, mechanical, and biological properties. We determined an optimal set of crosslinking conditions under visible light irradiation, which may permit us to engineer a hydrogel with tailored properties essential for specific biomedical applications.

\section{Methods}

Materials. All chemicals were used as received from Sigma-Aldrich (St. Louis, MO) without further purification unless otherwise mentioned. No animals or human participant was involved in the study, and only the fibroblast cell line (kind gift of Dr. J. Jester, UC-Irvine) was used to perform biocompatibility studies.

Chemical synthesis of GelMA. To synthesize GelMA with a high functionalization degree, gelatin ( $10 \mathrm{~g}$, $300 \mathrm{~g}$ Bloom, type A) was dissolved in $100 \mathrm{~mL}$ PBS solution, followed by the addition of $8 \mathrm{~mL}$ methacrylate anhydride as previously described ${ }^{22}$. The reaction mixtures were stirred for $3 \mathrm{~h}$ at $50{ }^{\circ} \mathrm{C}$, diluted with $100 \mathrm{~mL}$ deionized (DI) water, and dialyzed for 7 days using a dialysis membrane with a molecular weight cut-off of $14 \mathrm{kDa}$ (Sigma-Aldrich). Next, the GelMA solution was freeze-dried for 5 days to obtain a foam-like GelMA precursor.

Preparation of GelMA pre-polymer solutions. Varying [EY]. To prepare GelMA solutions with various [EY], $1.00 \mathrm{~g}$ GelMA was dissolved in $3.1 \mathrm{~mL} \mathrm{PBS}$ at $45^{\circ} \mathrm{C}$. Then, the solution was mixed with $200 \mu \mathrm{L}$ of $25 \%$ $(\mathrm{w} / \mathrm{v}) \mathrm{TEOA}, 500 \mu \mathrm{L}$ of $5.0 \%(\mathrm{w} / \mathrm{v}) \mathrm{VC}$, and varying contents of EY $(1.0,2.0,4.0,10,20,40,100$, and $200 \mu \mathrm{L}$ of EY with the concentration of $25 \mathrm{mM}(0.173 \mathrm{~g} / 10 \mathrm{~mL}))$ in PBS in the dark, and addition of PBS to make $5.0 \mathrm{~mL}$ GelMA solution, and agitated at $45^{\circ} \mathrm{C}$.

Varying [TEOA]. To prepare GelMA solutions with varying [TEOA], $1.00 \mathrm{~g}$ GelMA was dissolved in $2.6 \mathrm{~mL}$ PBS at $45^{\circ} \mathrm{C}$. Then, the solution was mixed with $500 \mu \mathrm{L}$ of $5.0 \%(\mathrm{w} / \mathrm{v}) \mathrm{VC}, 10 \mu \mathrm{L}$ of EY $(25 \mathrm{mM})$, and varying TEOA contents $(10,20,40,100,200,400,800 \mu \mathrm{L}$ of TEOA $(25 \% \mathrm{w} / \mathrm{v})$ in PBS in the dark, followed by addition of extra PBS to make $5.0 \mathrm{~mL}$ GelMA solution, and agitated at $45^{\circ} \mathrm{C}$.

Varying [VC]. To prepare GelMA solutions with varying [VC], $1.00 \mathrm{~g}$ GelMA was dissolved in $2.6 \mathrm{~mL}$ PBS solution at $45^{\circ} \mathrm{C}$. Then, the solution was mixed with $10 \mu \mathrm{L}$ of EY $(25 \mathrm{mM})$, of $200 \mu \mathrm{L}$ of TEOA $(25 \% \mathrm{w} / \mathrm{v})$, and varying VC contents $(50,100,200$, and $500 \mu \mathrm{L}$ of $\mathrm{VC}(5 \% \mathrm{w} / \mathrm{v}), 0.05,0.1$, and $0.2 \mathrm{~g}$ of VC) in PBS solution in the dark, followed by addition of extra PBS to make $5.0 \mathrm{~mL}$ GelMA solution, and agitation at $45^{\circ} \mathrm{C}$.

Varying [GelMA]. To prepare GelMA with varying concentrations, different amount of GelMA $(0.5,0.75,1$, and $1.25 \mathrm{~g})$ was dissolved in $3.1 \mathrm{~mL}$ PBS at $45^{\circ} \mathrm{C}$. Then, the solution was mixed with $10 \mu \mathrm{L}$ of EY $(25 \mathrm{mM})$, of $200 \mu \mathrm{L}$ of TEOA $(25 \% \mathrm{w} / \mathrm{v})$, and $0.1 \mathrm{~g}$ of VC in PBS in the dark conditions, followed by the addition of extra PBS to make $5.0 \mathrm{~mL}$ GelMA solution, and agitation at $45^{\circ} \mathrm{C}$.

Crosslinking conditions. After preparing the reaction mixtures as described above, the prepared solutions were carefully transferred to an appropriate mold and crosslinked for varying times $(0.5,1,2,4,7$, and $10 \mathrm{~min})$ using our hand-made visible light source (LED with a wavelength of 490-510 nm and the intensity of $20 \mathrm{~mW} /$ $\mathrm{cm}^{2}$ ).

Mechanical characterization. Mechanical properties studies were conducted using a mechanical tester (Instron mechanical tester; Norwood, MA) as previously described ${ }^{4,56}$. For tensile tests, dumbbell-shaped hydro- 
gels were prepared and stretched at a rate of $2 \mathrm{~mm} / \mathrm{min}$ until rupture, and the stress was recorded as a function of the strain. The tensile modulus was calculated from linear derivatives of the stress-strain plots at $0-60 \%$ strain $[\mathrm{n}=4]$. For the compression tests, disk-shaped samples were used and compressed with a crosshead speed of $0.5 \mathrm{~mm} / \mathrm{min}$ until the maximum stress of $0.6 \mathrm{MPa}^{4,57}$. The compressive stress was recorded as a function of the strain. The compressive modulus for each sample was determined from the linear derivatives of the stress-strain curves at $0-10 \%$ strain $[\mathrm{n}=4]$. Lap Shear strength of GelMA hydrogels was measured using a modified lap shear test according to ASTM F2255-05 standard lap shear test. First, a glass slide $(10 \times 40 \mathrm{~mm}$ diameter $)$ was functionalized with 3-(trimethoxysilyl)propyl methacrylate as previously described ${ }^{58}$. Then, $20 \mu \mathrm{L}$ of GelMA precursor solution was transferred on one slide and the other slide was carefully placed on the GelMA solution, and photo-crosslinked by visible light. The shear strengths of the samples were then determined using a mechanical tester with a crosshead speed of $1 \mathrm{~mm} / \mathrm{min}$, and the shear strengths of the GelMA were recorded at the detachment point $[n=4]$.

In vitro biodegradation. Enzymatic degradation of GelMA hydrogels was determined using collagenase from Clostridium histolyticum, as previously reported ${ }^{7}$. Briefly, we first generated disc-shape constructs, rinsed them with water, and placed them in a solution containing collagenase $(40 \mu \mathrm{g} / \mathrm{mL})$ in Tris- $\mathrm{HCl}$ buffer $(0.1 \mathrm{M}, \mathrm{pH}$ of 7.4), supplemented with $\mathrm{CaCl}_{2}(5 \mathrm{mM})$ and incubated at $37^{\circ} \mathrm{C}$. The collagenase solution was changed every $8 \mathrm{~h}$ and the hydrogel residue was carefully removed from the solution, rinsed with water, lyophilized, and then its dried mass at different time points $\left(W_{f}\right)$ was measured. $W_{\mathrm{i}}$ is the weight of discs after lyophilization. The degradation rate was calculated $[\mathrm{n}=4]$ using the following equation:Residualmass $(\%)=\frac{W_{f}}{W_{i}} \times 100$

Swelling ratio. To determine the swelling ratio, first, we generated disc-shape constructs, rinsed them with water, and blot dried them to obtain their initial wet weights $\left(W_{i}\right)$. Those discs were then immersed in PBS solution and incubated at $37^{\circ} \mathrm{C}$ for up to $24 \mathrm{~h}$. After predetermined periods $(2,4,12,24 \mathrm{~h})$, the swollen hydrogel samples were blot dried, and their swollen weights $\left(W_{s}\right)$ were measured. The swelling ratio $(S)$ for the hydrogels $[\mathrm{n}=4$ ] was calculated according to the following equation and plotted as a function of time. $S(\%)=\frac{\left(W_{s}-W_{i}\right)}{W_{i}} \times 100$

In vitro and ex vivo biocompatibility. Cell encapsulation ( $3 D$ culture). Fibroblast cells were trypsinized and resuspended $\left(2 \times 10^{5}\right.$ cells per $\left.\mathrm{mL}\right)$ in the hydrogel precursor $(10 \%$ GelMA solution with varying crosslinking concentrations as described above). Then, $20 \mu \mathrm{L}$ of the cell dispersion was transferred to polydimethylsiloxane (PDMS) mold (diameter $=8 \mathrm{~mm}$, thickness $=0.2 \mathrm{~mm}$ ) and exposed to photoirradiation for a varying period. Those cell-laden gels were immediately washed 3 times with PBS and incubated in $200 \mu \mathrm{L}$ culture medium (DMEM/F-12 50/50, $1 \times$ media (Corning, VA, USA) at $37^{\circ} \mathrm{C}^{59}$. For mesenchymal stems cells, after resuspending $\left(2 \times 10^{5}\right.$ cells per $\left.\mathrm{mL}\right)$ in the hydrogel precursor solution containing $(10 \%$ GelMA, $0.05 \mathrm{mM} \mathrm{EY,} \%$ (w/v) TEOA, and $1 \%(\mathrm{w} / \mathrm{v})$, the suspension $(20 \mu \mathrm{L})$ was transferred to PDMS mold and exposed to photoirradiation for a 4 and $10 \mathrm{~min}$. Those cell-laden gels were immediately washed 3 times with PBS and incubated in $200 \mu \mathrm{L}$ of an optimized serum-containing medium (catalog \# 02,100, Reachbio lab, Spokane, WA) at $37^{\circ} \mathrm{C}$.

Cell viability. The viability of cells was assessed using a commercial kit (LIVE/DEAD ${ }^{\text {tw }}$ viability/cytotoxicity kit, for mammalian cells, (Thermofisher Scientific; Cambridge, MA), as previously described ${ }^{60,61}$. Briefly, cells were double-stained with calcein acetoxymethyl and ethidium homodimer for $30 \mathrm{~min}$ and imaged by inverted fluorescent microscopy (Zeiss Axio Observer Z1; Thornwood, NY) with a 10X objective. The number of live and dead cells was quantified using the ImageJ software (NIH, Bethesda, Maryland), and the cell viability was assessed from the number of live cells divided by the total number of live and dead cells ${ }^{60}$. Three samples were used for each condition and four images were acquired from each sample.

AlamarBlue Assay. To assess the metabolic activity of the encapsulated cells in GelMA, we used a standard AlamarBlue assay as explained elsewhere ${ }^{62}$. Briefly, cell-laden gels were incubated at $37^{\circ} \mathrm{C}$ and $5 \% \mathrm{CO}_{2}$ conditions for up to 7 days. The AlamarBlue test was performed on days 1, 4, and 7 after cell seeding. At each time point, the tissue culture media was replaced with fresh media $(200 \mu \mathrm{L})$ containing resazurin sodium salt $(0.004 \%$ $\mathrm{w} / \mathrm{v})$ and incubated for $2 \mathrm{~h}$. Afterward, the media $(100 \mu \mathrm{L})$ was transferred into a new 96 well plate and read on a BioTek plate reader (Synergy 2, BioTek Instruments; Winooski, VT) at 530/25 nm for excitation and $600 / 25 \mathrm{~nm}$ for emission and corrected with the fluorescence of GelMA discs incubated without cells. Four samples for each group and 3-time points were tested.

Data analysis. Mechanical properties, swelling ratios, enzymatic degradation, and cytotoxicity data as a function of reaction conditions were plotted using OriginLab 2018, and the data fitting process was performed using exponential growth and decay and sigmoidal functions as described in the main text.

Statistical analysis. One-way ANOVA with Tukey comparison test on GraphPad Prism Software (GraphPad Software version 9.2.0, CA, USA) was used to compare cell viability between groups. A value of $p \leq 0.05$ was considered statistically significant. n.s., ${ }^{*}{ }^{* *},{ }^{* *}$, and ${ }^{* * * *}$ represent $p>0.05, p \leq 0.05, p \leq 0.01, p \leq 0.001$ and $p \leq 0.0001$, respectively.

Received: 18 July 2021; Accepted: 18 November 2021

Published online: 02 December 2021 


\section{References}

1. Silva, R., Fabry, B. \& Boccaccini, A. R. Fibrous protein-based hydrogels for cell encapsulation. Biomaterials 35, 6727-6738. https:// doi.org/10.1016/j.biomaterials.2014.04.078 (2014).

2. Leijten, J. et al. Spatially and temporally controlled hydrogels for tissue engineering. Mater. Sci. Eng. R Rep. 119, 1-35. https://doi. org/10.1016/j.mser.2017.07.001 (2017).

3. Trujillo-de Santiago, G. et al. Ocular adhesives: Design, chemistry, crosslinking mechanisms, and applications. Biomaterials 197, 345-367. https://doi.org/10.1016/j.biomaterials.2019.01.011 (2019).

4. Sharifi, S. et al. Tuning gelatin-based hydrogel towards bioadhesive ocular tissue engineering applications. Bioact. Mater. 6, 39473961. https://doi.org/10.1016/j.bioactmat.2021.03.042 (2021).

5. Yue, K. et al. Synthesis, properties, and biomedical applications of gelatin methacryloyl (GelMA) hydrogels. Biomaterials 73, 254-271 (2015).

6. Camci-Unal, G., Cuttica, D., Annabi, N., Demarchi, D. \& Khademhosseini, A. Synthesis and characterization of hybrid hyaluronic acid-gelatin hydrogels. Biomacromol 14, 1085-1092. https://doi.org/10.1021/bm3019856 (2013).

7. Chen, Y. C. et al. Functional human vascular network generated in photocrosslinkable gelatin methacrylate hydrogels. Adv. Funct. Mater. 22, 2027-2039. https://doi.org/10.1002/adfm.201101662 (2012).

8. Nikkhah, M. et al. Directed endothelial cell morphogenesis in micropatterned gelatin methacrylate hydrogels. Biomaterials 33, 9009-9018. https://doi.org/10.1016/j.biomaterials.2012.08.068 (2012).

9. Shirahama, H., Lee, B. H., Tan, L. P. \& Cho, N. J. Precise tuning of facile one-pot gelatin methacryloyl (GelMA) synthesis. Sci. Rep. 6, 31036. https://doi.org/10.1038/srep31036 (2016).

10. Annabi, N. et al. Engineered cell-laden human protein-based elastomer. Biomaterials 34, 5496-5505. https://doi.org/10.1016/j. biomaterials.2013.03.076 (2013).

11. Zhang, Y. N. et al. A highly elastic and rapidly crosslinkable elastin-like polypeptide-based hydrogel for biomedical applications. Adv. Funct. Mater. 25, 4814-4826. https://doi.org/10.1002/adfm.201501489 (2015).

12. Andreopoulos, F. M., Beckman, E. J. \& Russell, A. J. Light-induced tailoring of PEG-hydrogel properties. Biomaterials 19, 13431352. https://doi.org/10.1016/s0142-9612(97)00219-6 (1998).

13. Lang, N. et al. A blood-resistant surgical glue for minimally invasive repair of vessels and heart defects. Sci. Transl. Med. 6, $218 \mathrm{ra} 216$. https://doi.org/10.1126/scitranslmed.3006557 (2014).

14. Pereira, M. J. et al. A highly tunable biocompatible and multifunctional biodegradable elastomer. Adv. Mater. 25, $1209-1215$. https://doi.org/10.1002/adma.201203824 (2013).

15. Goodsell, D. S. The molecular perspective: ultraviolet light and pyrimidine dimers. Stem Cells 19, 348-349. https://doi.org/10. 1634/stemcells.19-4-348 (2001).

16. Kielbassa, C., Roza, L. \& Epe, B. Wavelength dependence of oxidative DNA damage induced by UV and visible light. Carcinogenesis 18, 811-816. https://doi.org/10.1093/carcin/18.4.811 (1997).

17. Sato, R., Harada, R. \& Shigeta, Y. Theoretical analyses on a flipping mechanism of UV-induced DNA damage. Biophys. Physicobiol. 13, 311-319. https://doi.org/10.2142/biophysico.13.0_311 (2016).

18. Fedorovich, N. E. et al. The effect of photopolymerization on stem cells embedded in hydrogels. Biomaterials 30, 344-353. https:// doi.org/10.1016/j.biomaterials.2008.09.037 (2009).

19. Monteiro, N. et al. Photopolymerization of cell-laden gelatin methacryloyl hydrogels using a dental curing light for regenerative dentistry. Dent. Mater. 34, 389-399. https://doi.org/10.1016/j.dental.2017.11.020 (2018).

20. Nakayama, Y., Kameo, T., Ohtaka, A. \& Hirano, Y. Enhancement of visible light-induced gelation of photocurable gelatin by addition of polymeric amine. J. Photochem. Photobiol. A 177, 205-211. https://doi.org/10.1016/j.jphotochem.2005.05.030 (2006).

21. Elisseeff, J. et al. Transdermal photopolymerization for minimally invasive implantation. Proc. Natl. Acad. Sci. USA 96, 3104-3107. https://doi.org/10.1073/pnas.96.6.3104 (1999).

22. Shirzaei Sani, E. et al. Sutureless repair of corneal injuries using naturally derived bioadhesive hydrogels. Sci. Adv. 5, eaav1281 (2019).

23. Tomal, W. \& Ortyl, J. Water-soluble photoinitiators in biomedical applications. Polymers 12, 1073 (2020).

24. Wäldchen, S., Lehmann, J., Klein, T., van de Linde, S. \& Sauer, M. Light-induced cell damage in live-cell super-resolution microscopy. Sci. Rep. UK 5, 15348. https://doi.org/10.1038/srep15348 (2015).

25. Marek, V. et al. Blue light exposure in vitro causes toxicity to trigeminal neurons and glia through increased superoxide and hydrogen peroxide generation. Free Radical Biol. Med. 131, 27-39. https://doi.org/10.1016/j.freeradbiomed.2018.11.029 (2019).

26. Noshadi, I. et al. In vitro and in vivo analysis of visible light crosslinkable gelatin methacryloyl (GelMA) hydrogels. Biomater. Sci. 5, 2093-2105. https://doi.org/10.1039/c7bm00110j (2017).

27. Hao, Y., Shih, H., Munoz, Z., Kemp, A. \& Lin, C. C. Visible light cured thiol-vinyl hydrogels with tunable degradation for 3D cell culture. Acta Biomater. 10, 104-114. https://doi.org/10.1016/j.actbio.2013.08.044 (2014).

28. Bahney, C. S. et al. Visible light photoinitiation of mesenchymal stem cell-laden bioresponsive hydrogels. Eur. Cell Mater. 22, 43-55; discussion 55. doi:https://doi.org/10.22203/ecm.v022a04 (2011).

29. Sharifi, S. et al. Photo-cross-linked gelatin glycidyl methacrylate/N-vinylpyrrolidone copolymeric hydrogel with tunable mechanical properties for ocular tissue engineering applications. ACS Appl. Bio Mater. 4, 7682-7691. https://doi.org/10.1021/acsabm.1c009 05 (2021).

30. Williams, C. G., Malik, A. N., Kim, T. K., Manson, P. N. \& Elisseeff, J. H. Variable cytocompatibility of six cell lines with photoinitiators used for polymerizing hydrogels and cell encapsulation. Biomaterials 26, 1211-1218. https://doi.org/10.1016/j.biomateria ls.2004.04.024 (2005).

31. Gurski, L. A., Petrelli, N. J., Jia, X. \& Farach-Carson, M. C. 3D matrices for anti-cancer drug testing and development. Oncol. Issues 25, 20-25. https://doi.org/10.1080/10463356.2010.11883480 (2017).

32. Akbari, A. et al. Free and hydrogel encapsulated exosome-based therapies in regenerative medicine. Life Sci 249, 117447. https:// doi.org/10.1016/j.lfs.2020.117447 (2020).

33. Zhang, Y. S. et al. 3D Bioprinting for tissue and organ fabrication. Ann. Biomed. Eng. 45, 148-163. https://doi.org/10.1007/s10439016-1612-8 (2017).

34. Sharifi, R., Samaraweera, M., Gascon, J. A. \& Papadimitrakopoulos, F. Thermodynamics of the quasi-epitaxial flavin assembly around various-chirality carbon nanotubes. J. Am. Chem. Soc. 136, 7452-7463. https://doi.org/10.1021/ja502714z (2014).

35. White, T. J., Liechty, W. B. \& Guymon, C. A. The influence ofN-vinyl pyrrolidone on polymerization kinetics and thermo-mechanical properties of crosslinked acrylate polymers. J. Polym. Sci., Part A Polym. Chem. 45, 4062-4073. https://doi.org/10.1002/pola. 22173 (2007).

36. Aguirre-Soto, A., Kim, S., Kaastrup, K. \& Sikes, H. D. On the role of N-vinylpyrrolidone in the aqueous radical-initiated copolymerization with PEGDA mediated by eosin Y in the presence of O2. Polym. Chem. 10, 926-937. https://doi.org/10.1039/c8py0 1459k (2019).

37. Sperling, L. H. Introduction to Physical Polymer Science (Wiley, 2015).

38. Nemir, S. \& West, J. L. Synthetic materials in the study of cell response to substrate rigidity. Ann. Biomed. Eng. 38, 2-20. https:// doi.org/10.1007/s10439-009-9811-1 (2010). 
39. Bott, K. et al. The effect of matrix characteristics on fibroblast proliferation in 3D gels. Biomaterials 31, 8454-8464. https://doi. org/10.1016/j.biomaterials.2010.07.046 (2010).

40. Peyton, S. R., Raub, C. B., Keschrumrus, V. P. \& Putnam, A. J. The use of poly(ethylene glycol) hydrogels to investigate the impact of ECM chemistry and mechanics on smooth muscle cells. Biomaterials 27, 4881-4893. https://doi.org/10.1016/j.biomaterials. 2006.05.012 (2006).

41. Assmann, A. et al. A highly adhesive and naturally derived sealant. Biomaterials 140, 115-127. https://doi.org/10.1016/j.bioma terials.2017.06.004 (2017).

42. Elbert, D. L. \& Hubbell, J. A. Conjugate addition reactions combined with free-radical cross-linking for the design of materials for tissue engineering. Biomacromol 2, 430-441. https://doi.org/10.1021/bm0056299 (2001).

43. Turturro, M. V., Sokic, S., Larson, J. C. \& Papavasiliou, G. Effective tuning of ligand incorporation and mechanical properties in visible light photopolymerized poly(ethylene glycol) diacrylate hydrogels dictates cell adhesion and proliferation. Biomed Mater 8, 025001. https://doi.org/10.1088/1748-6041/8/2/025001 (2013).

44. Odian, G. in Principles of Polymerization 198-349 (2004).

45. Su, W.-F. in Principles of Polymer Design and Synthesis Lecture Notes in Chemistry Ch. Chapter 7, 137-183 (Springer, 2013).

46. Holloway, J. L., Ma, H., Rai, R. \& Burdick, J. A. Modulating hydrogel crosslink density and degradation to control bone morphogenetic protein delivery and in vivo bone formation. J. Control Release 191, 63-70. https://doi.org/10.1016/j.jconrel.2014.05.053 (2014).

47. Zhao, F., Bi, W. \& Zhao, S. Influence of crosslink density on mechanical properties of natural rubber vulcanizates. J. Macromol. Sci. Part B 50, 1460-1469. https://doi.org/10.1080/00222348.2010.507453 (2011).

48. Lee, K. Y. et al. Controlling mechanical and swelling properties of alginate hydrogels independently by cross-linker type and cross-linking density. Macromolecules 33, 4291-4294. https://doi.org/10.1021/ma9921347 (2000).

49. Knaak, J. B., Leung, H.-W., Stott, W. T., Busch, J. \& Bilsky, J. in Reviews of Environmental Contamination and Toxicology: Continuation of Residue Reviews (ed George W. Ware) 1-86 (Springer, 1997).

50. McGrath, J. E. Chain reaction polymerization. J. Chem. Educ. 58, 844. https://doi.org/10.1021/ed058p844 (1981)

51. Zhang, Y., Won, C.-Y. \& Chu, C.-C. Synthesis and characterization of biodegradable hydrophobic-hydrophilic hydrogel networks with a controlled swelling property. J. Polym. Sci. Part A Polym. Chem. 38, 2392-2404. https://doi.org/10.1002/1099-0518(20000 701)38:13\%3c2392::Aid-pola120\%3e3.0.Co;2-p (2000).

52. Vihola, H., Laukkanen, A., Valtola, L., Tenhu, H. \& Hirvonen, J. Cytotoxicity of thermosensitive polymers poly(N-isopropylacrylamide), poly(N-vinylcaprolactam) and amphiphilically modified poly(N-vinylcaprolactam). Biomaterials 26, 3055-3064. https:// doi.org/10.1016/j.biomaterials.2004.09.008 (2005).

53. Sigma-Aldrich. Safety Data Sheet, <https://www.chemblink.com/MSDS/MSDSFiles/2235-00-9_Sigma-Aldrich.pdf> (2018).

54. Nichol, J. W. et al. Cell-laden microengineered gelatin methacrylate hydrogels. Biomaterials 31, 5536-5544. https://doi.org/10. 1016/j.biomaterials.2010.03.064 (2010).

55. Bianco, P. et al. The meaning, the sense and the significance: Translating the science of mesenchymal stem cells into medicine. Nat. Med. 19, 35-42. https://doi.org/10.1038/nm.3028 (2013).

56. ASTM. in Standard Test Method for Tensile Properties of Plastics (ASTM International, 2014).

57. Prebensen, C. et al. High MIP-1beta levels in plasma predict long-term immunological nonresponse to suppressive antiretroviral therapy in HIV infection. J. Acquir. Immune Defic. Syndr. 1999(69), 395-402. https://doi.org/10.1097/qai.0000000000000617 (2015).

58. Garoff, H. \& Ansorge, W. Improvements of DNA sequencing gels. Anal. Biochem. 115, 450-457. https://doi.org/10.1016/00032697(81)90031-2(1981).

59. Sharifi, S. et al. Sputter deposition of titanium on poly(methyl methacrylate) enhances corneal biocompatibility. Transl. Vis. Sci. Technol. 9, 41. https://doi.org/10.1167/tvst.9.13.41 (2020).

60. Sharifi, R. et al. Covalent functionalization of pmma surface with 1-3,4-dihydroxyphenylalanine (L-DOPA) to enhance its biocompatibility and adhesion to corneal tissue. Adv. Mater. Interfaces 7, 1900767 (2020).

61. Sharifi, S. et al. Electron beam sterilization of poly(methyl methacrylate)-physicochemical and biological aspects. Macromol Biosci 21, e2000379. https://doi.org/10.1002/mabi.202000379 (2021).

62. Gonzalez-Andrades, M. et al. Improving the practicality and safety of artificial corneas: Pre-assembly and gamma-rays sterilization of the Boston Keratoprosthesis. Ocul. Surf. 16, 322-330. https://doi.org/10.1016/j.jtos.2018.04.002 (2018).

\section{Acknowledgements}

This work was supported by NIH K99 EY030553, and by EBAA/ Richard Lindstrom Research Grant (530720). This work was performed in part at the Center for Nanoscale Systems (CNS), Harvard University, a member of the National Nanotechnology Coordinated Infrastructure Network (NNCI), which is supported by the National Science Foundation under NSF award no. 1541959.

\section{Author contributions}

S.S. is responsible for the generation of the concept, study design, data analysis, and preparation of the manuscript. H.S. performed mechanical tests. S.S., H.S., A.A., and J.C. discussed the results, and revised the manuscript. J.C. corrected the manuscript for publication. All authors contributed to the final manuscript preparation and have approved the same.

\section{Funding}

National Eye Institute, K99 EY030553,Richard Lindstrom Research Grant 530720.

\section{Competing interests}

The authors declare no competing interests.

\section{Additional information}

Supplementary Information The online version contains supplementary material available at https://doi.org/ 10.1038/s41598-021-02830-x.

Correspondence and requests for materials should be addressed to S.S.

Reprints and permissions information is available at www.nature.com/reprints.

Publisher's note Springer Nature remains neutral with regard to jurisdictional claims in published maps and institutional affiliations. 
(c) (i) Open Access This article is licensed under a Creative Commons Attribution 4.0 International cc) License, which permits use, sharing, adaptation, distribution and reproduction in any medium or format, as long as you give appropriate credit to the original author(s) and the source, provide a link to the Creative Commons licence, and indicate if changes were made. The images or other third party material in this article are included in the article's Creative Commons licence, unless indicated otherwise in a credit line to the material. If material is not included in the article's Creative Commons licence and your intended use is not permitted by statutory regulation or exceeds the permitted use, you will need to obtain permission directly from the copyright holder. To view a copy of this licence, visit http://creativecommons.org/licenses/by/4.0/.

(C) The Author(s) 2021 